\title{
A Heuristic Solution to the Transformer Manufacturing Cost Optimization Problem
}

\author{
Pavlos S. Georgilakis ${ }^{1}$, Marina A. Tsili ${ }^{2}$ and Athanassios T. Souflaris ${ }^{3}$ \\ ${ }^{1}$ Department of Production Engineering \& Management, Technical University of Crete, \\ GR-73100, Chania, Greece, \\ e-mail :pgeorg@dpem.tuc.gr \\ ${ }^{2}$ Faculty of Electrical \& Computer Engineering, National Technical University of Athens, \\ GR-15780, Athens, Greece, \\ e-mail:mtsilil@central.ntua.gr \\ ${ }^{3}$ Schneider Electric AE, Elvim Plant, GR-32011, Inofyta, Viotia, Greece, \\ e-mail : thanassis.souflaris@gr.schneider-electric.com
}

\begin{abstract}
The aim of the transformer design is to completely obtain the dimensions of all the parts of the transformer based on the given specification, using available materials economically in order to achieve lower cost, lower weight, reduced size and better operating performance. In this paper, a heuristic optimization method is proposed aiming at designing the transformer so as to meet the specification with the minimum manufacturing cost. Results from the application of the proposed methodology demonstrate the effectiveness and practicality of this approach.
\end{abstract}

\section{Introduction}

The aim of the transformer design is to completely obtain the dimensions of all the parts of the transformer to furnish these data to the manufacturer. The transformer design should be carried out based on the given specification, using available materials economically in order to achieve lower cost, lower weight, reduced size and better operating performance [1]. The transformer design is worked out by using various methods based on accumulated experience realized in different formulae, equations, tables and charts. The transformer design methods vary between the several transformer manufacturers. While designing a transformer, much emphasis should be placed on lowering its manufacturing cost by saving the materials and reducing to a minimum labor consuming operations in its manufacture. The design should be satisfactory with respect to electric strength, mechanical endurance, dynamic and thermal resistances of windings in the event of shortcircuit. In this paper, a heuristic optimization method is proposed aiming at designing the transformer so as to meet the specification with the minimum manufacturing cost.

\section{Transformer specifications}

The transformer manufacturing is based on the international standards and also on customer needs (technical specifications). Tolerances should be applied to certain rated transformer quantities when they are the subjects of manufacturer's guarantees [2].

\section{Transformer manufacturing cost optimization method}

The power transformers considered in this paper are three-phase, wound core and their magnetic circuit is of shell type. The transformer manufacturing cost is equal to the sum of the total materials cost plus the labor cost. The heuristic optimization method aims at finding the so-called technical and economical optimum transformer, i.e. the transformer with the minimum manufacturing cost. The proposed optimization method is implemented with the help of a suitable computer program, which uses 134 input parameters in order to make the transformer program as parametric as possible. The computer program allows many variations in certain design variables, which are called solution loop variables. These variations permit the investigation of enough candidate solutions. For each one of the candidate solutions, it is checked if all the specifications (limits) are satisfied, and if they are satisfied, the manufacturing cost is estimated and the solution is characterized as acceptable. On the other hand, the candidate solutions that violate the specification are characterized as nonacceptable solutions. Finally, from the acceptable solutions, the transformer with the minimum manufacturing cost is selected, which is the technical and economical optimum transformer. 


\section{Results and discussion}

The proposed transformer manufacturing cost optimization method is already applied in a transformer manufacturing industry. The efficiency of the proposed algorithm is presented through an actual design example of a transformer with rated power $160 \mathrm{kVA}$, rated voltages $20 / .4 \mathrm{kV}$, vector group Dyn 11 , frequency $50 \mathrm{~Hz}$. The constraints related to transformer losses and short-circuit impedance $\left(\mathrm{U}_{\mathrm{k}}\right)$ are the following: maximum copper $(\mathrm{Cu})$ losses $3000 \mathrm{~W}$, maximum iron $(\mathrm{Fe})$ losses $380 \mathrm{~W}$, minimum $\mathrm{U}_{\mathrm{k}} 3.6 \%$ and maximum $\mathrm{U}_{\mathrm{k}} 4.4 \%$.

The transformer designer gives appropriate and enough alternative values to the solution loop variables and the computer program considers 8000 candidate solutions. From these 8000 candidate solutions, 2025 are acceptable solutions, while the rest 5975 are rejected (non-acceptable) solutions, since they violate the specification, for example they violate at least one of the constraints for Cu losses, Fe losses, or for $U_{k}$.

Table I presents the first 5 (cheapest) and the last 5 (more expensive) solutions from the total 2025 accepted solutions. It is noticed that the cheapest solution (technical and economical optimum transformer) costs $€ 1121.99$ and the most expensive solution costs $€ 1418.32$. Namely, the optimum technical and economical solution is $26.4 \%$ cheaper than the most expensive solution.

The technical and economical optimum transformer is the transformer number 1 of Table I, which has the following technical characteristics (not listed in Table I): copper losses $2838.8 \mathrm{~W}$, iron losses $364.1 \mathrm{~W}$, shortcircuit impedance $4.03 \%$, so the technical and economical optimum solution satisfies the constraints for losses and short-circuit impedance. As it can be seen from Table I, the manufacturing cost of the optimum transformer is $€ 1121.99$, the height of core window (G) is $225 \mathrm{~mm}$, the width of core leg (D) is $190 \mathrm{~mm}$, the low voltage (LV) coil has 32 turns, the rated magnetic induction is 17500 Gauss, the LV conductor is sheet with width $207 \mathrm{~mm}$ and thickness $0.3 \mathrm{~mm}$ and the high voltage (HV) conductor is wire with diameter $1 \mathrm{~mm}$.

Sensitivity analysis can be carried out in order to check if the optimum solution can be further improved. For example, if we consider only the variation of the cross-section area (CSA) of the low voltage coil and if we execute again the transformer optimization program, we obtain the results of Table II, from which it is concluded that the optimum solution is the same with the one already found in Table I. Moreover, from Table II, it is concluded that, in general, the copper losses are decreased with the increase of the cross-section area of the low voltage coil.

Table I: Acceptable solutions

\begin{tabular}{|c|c|c|c|c|c|c|c|c|c|c|}
\hline \multicolumn{2}{|c|}{ Number } & Cost $(€)$ & $G(\mathrm{~mm})$ & $D(\mathrm{~mm})$ & LV turns & B (Gauss) & \multicolumn{2}{|c|}{ LV conductor } & \multicolumn{2}{|c|}{ HV conductor } \\
\hline \multicolumn{2}{|c|}{1} & 1121.99 & 225 & 190 & 32 & 17500 & \multicolumn{2}{|c|}{207 x 0.3} & \multicolumn{2}{|c|}{1} \\
\hline \multicolumn{2}{|c|}{2} & 1123.44 & 230 & 190 & 33 & 17500 & \multicolumn{2}{|c|}{$212 \times 0.3$} & \multicolumn{2}{|c|}{1} \\
\hline \multicolumn{2}{|c|}{3} & 1123.77 & 225 & 220 & 30 & 17500 & \multicolumn{2}{|c|}{207 x 0.3} & \multicolumn{2}{|c|}{1} \\
\hline \multicolumn{2}{|c|}{4} & 1123.95 & 230 & 190 & 31 & 17500 & \multicolumn{2}{|c|}{$212 \times 0.3$} & \multicolumn{2}{|c|}{1} \\
\hline \multicolumn{2}{|c|}{5} & 1124.87 & 225 & 237 & 30 & 17500 & \multicolumn{2}{|c|}{$207 \times 0.3$} & \multicolumn{2}{|c|}{1} \\
\hline \multicolumn{2}{|c|}{2021} & 1401.83 & 180 & 152 & 26 & 13000 & \multicolumn{2}{|c|}{$162 \times 0.4$} & \multicolumn{2}{|c|}{1} \\
\hline \multicolumn{2}{|c|}{2022} & 1406.16 & 195 & 152 & 27 & 13000 & \multicolumn{2}{|c|}{$177 \times 0.4$} & \multicolumn{2}{|c|}{1} \\
\hline \multicolumn{2}{|c|}{2023} & 1408.16 & 190 & 152 & 26 & 13000 & 172 & 0.4 & 1 & \\
\hline 202 & & 1409.87 & 185 & 152 & 26 & 13000 & 167 & 0.4 & 1 & \\
\hline 202 & & 1418.32 & 195 & 152 & 26 & 13000 & 177 & 0.4 & 1 & \\
\hline & & & I & riotion $f$ & sotion & a (CSA) of low & voltage (LV & coil & & \\
\hline Input & ariable & & & & & & Output val & ables & & \\
\hline $\begin{array}{c}\mathrm{G} \\
(\mathrm{mm})\end{array}$ & $\begin{array}{c}\mathrm{D} \\
(\mathrm{mm})\end{array}$ & $\begin{array}{l}\mathrm{LV} \\
\text { turns }\end{array}$ & $\begin{array}{c}\mathrm{B} \\
\text { (Gauss) }\end{array}$ & $\begin{array}{c}\mathrm{LV} \\
\text { conductor }\end{array}$ & $\begin{array}{c}\text { LV CSA } \\
\left(\mathrm{mm}^{2}\right)\end{array}$ & $\begin{array}{c}\mathrm{HV} \\
\text { conductor }\end{array}$ & $\begin{array}{c}\text { Cu losses } \\
(\mathrm{W})\end{array}$ & $\begin{array}{c}\text { Fe losses } \\
\text { (W) }\end{array}$ & $\begin{array}{c}\mathrm{U}_{\mathrm{k}} \\
(\%)\end{array}$ & Cost $(€)$ \\
\hline 225 & 190 & 32 & 17500 & $207 \times 0.30$ & 62.10 & 1 & 2838.8 & 364.1 & 4.03 & 1121.99 \\
\hline 225 & 190 & 32 & 17500 & $207 \times 0.31$ & 64.17 & 1 & 2804.4 & 364.1 & 4.04 & 1124.39 \\
\hline 225 & 190 & 32 & 17500 & $207 \times 0.32$ & 66.24 & 1 & 2772.3 & 364.1 & 4.06 & 1128.05 \\
\hline 225 & 190 & 32 & 17500 & $207 \times 0.33$ & 68.31 & 1 & 2742.4 & 364.1 & 4.07 & 1130.48 \\
\hline 225 & 190 & 32 & 17500 & $207 \times 0.34$ & 70.38 & 1 & 2714.5 & 368.4 & 4.08 & 1140.64 \\
\hline 225 & 190 & 32 & 17500 & $207 \times 0.35$ & 72.45 & 1 & 2688.4 & 368.4 & 4.10 & 1144.33 \\
\hline
\end{tabular}

\section{References}

[1] V.N. Mittle and A. Mittal, Design of Electrical Machines, $4^{\text {th }}$ Edition. Standard Publishers Distributors, Delhi, 1996.

[2] IEC 60076-1, "Power transformers - Part 1: General," 2000.

[3] Z. K. Papazacharopoulos, K. V. Tatis, A. G. Kladas, and S. N. Manias, "Dynamic Model for Harmonic Induction Motor Analysis Determined by Finite Elements”, IEEE Trans. on En. Conversion, Vol. 19, No. 1, 2004, pp. 102-108.

[4] Z. Papazacharopoulos, A. Kladas, S. Manias and J. Tegopoulos, "High fidelity model for induction motor drive including iron losses due to PWM waveforms", International Journal on Applied Electromagnetics and Mechanics, Vol. 12, no 3, pp.44-48, IOS Press, 2001. 